\title{
Magnetic Resonance Arthrography of Lesser Metatarsophalangeal Joints in Patients with Rheumatoid Arthritis: Relationship to Clinical, Biomechanical, and Radiographic Variables
}

\author{
HEIDI J. SIDDLE, RICHARD J. HODGSON, PHILIP O'CONNOR, ANDREW J. GRAINGER, ANTHONY C. REDMOND, \\ RICHARD J. WAKEFIELD, ELIZABETH M.A. HENSOR, and PHILIP S. HELLIWELL
}

\begin{abstract}
Objective. Our exploratory study of painful lesser metatarsophalangeal (MTP) joints in patients with rheumatoid arthritis (RA) primarily aimed to compare the clinical, biomechanical, and plain radiography findings with magnetic resonance (MR) arthrography findings. Our secondary aim was to compare standard unenhanced MR with MR arthrography in imaging the lesser MTP joints in RA. Methods. In 15 patients with RA, the more symptomatic forefoot was imaged using 3T MR imaging. Proton density fat-suppressed images were acquired through the lesser MTP joints prior to arthrography. Under ultrasound guidance, contrast agent was injected into 2 lesser MTP joints. T1-weighted fat-suppressed sequences were subsequently acquired. The MR images were read by 2 musculoskeletal radiologists and consensus was reached. Spearman's correlation coefficient was used to assess the association between abnormalities seen on MR arthrography and the clinical, biomechanical, and plain radiography findings.

Results. MR arthrography demonstrated pathology at 18 of 28 lesser MTP joints (64\%) examined in patients with RA. MR arthrography abnormalities were associated with RA disease duration, forefoot deformity, Larsen score, subluxation, and peak plantar pressure. Unenhanced MR had a sensitivity of $78 \%$ and specificity of $90 \%$ for detecting pathology compared to MR arthrography.

Conclusion. Capsule and plantar plate pathology occurs in the painful forefoot of patients with RA and is associated with features of disease and deformity at the lesser MTP joints. Compared with MR arthrography, standard MR imaging was highly specific and moderately sensitive for diagnosing lesser MTP joint pathology in patients with RA. (First Release Aug 1 2012; J Rheumatol 2012;39:1786-91; doi:10.3899/jrheum.120392)
\end{abstract}

\section{Key Indexing Terms:}

RHEUMATOID ARTHRITIS FOOT MAGNETIC RESONANCE IMAGING

From the Division of Rheumatic and Musculoskeletal Disease, Leeds Institute of Molecular Medicine, University of Leeds; National Institute for Health Research (NIHR) Leeds Musculoskeletal Biomedical Research Unit, and Department of Radiology, Leeds Teaching Hospitals National Health Service (NHS) Trust, Chapel Allerton Hospital, Leeds, UK. Supported by Arthritis Research UK (grant 18007) and the NIHR. H.J. Siddle, BSc (Hons), MSc, Division of Rheumatic and Musculoskeletal Disease, Leeds Institute of Molecular Medicine, University of Leeds, Chapel Allerton Hospital; R.J. Hodgson, BM, FRCR, PhD, NIHR Leeds Musculoskeletal Biomedical Research Unit, Leeds Teaching Hospitals NHS Trust, Chapel Allerton Hospital; P. O'Connor, BM, BS, MRCP, FRCR, FFSEM(UK); A.J. Grainger, BM, BS, MRCP, FRCR, NIHR Leeds Musculoskeletal Biomedical Research Unit and Department of Radiology, Leeds Teaching Hospitals NHS Trust, Chapel Allerton Hospital;

A.C. Redmond, MSc, PhD; R.J. Wakefield, BM, FRCP, MD, Division of Rheumatic and Musculoskeletal Disease, Leeds Institute of Molecular Medicine, University of Leeds, and NIHR Leeds Musculoskeletal Biomedical Research Unit, Leeds Teaching Hospitals NHS Trust, Chapel Allerton Hospital; E.M.A. Hensor, PhD; P.S. Helliwell, MA, DM, FRCP, PhD, Division of Rheumatic and Musculoskeletal Disease, Leeds Institute of Molecular Medicine, University of Leeds, Chapel Allerton Hospital. Address correspondence to H.J. Siddle, Division of Rheumatic and Musculoskeletal Disease, Leeds Institute of Molecular Medicine, University of Leeds, 2nd Floor, Chapel Allerton Hospital, Chapeltown Road, Leeds, LS7 4SA, UK. E-mail: h.siddle@leeds.ac.uk

Full Release Article. For details see Reprints/Permissions at jrheum.org Accepted for publication June 18, 2012.

\section{METATARSOPHALANGEAL JOINT ARTHROGRAPHY}

Pain and deformity at the metatarsophalangeal (MTP) joints in patients with rheumatoid arthritis (RA) are commonly reported despite improved systemic disease control ${ }^{1}$. Forefoot synovitis of the MTP joints is a significant feature of both early and active disease, leading to deformities such as hallux valgus, forefoot widening, hammer and claw toes, and subluxation and dislocation of the joints and plantar bursae $^{2}$. Inflammation of the synovium may produce separation of the toes known as the daylight sign, resulting in stretching and weakening of the capsule and loss of integrity of the stabilizing structures, such as the collateral ligaments and plantar plate ${ }^{3,4}$. As a consequence, it is hypothesized that subluxation followed by dislocation of the MTP joints occurs, resulting in the painful sensation often described as walking on pebbles.

Cadaveric studies of the feet in patients with RA have suggested that forefoot deformities might result from a failure of the complex ligamentous system around the MTP joints and the dynamic effect of displacement of the plantar plate ${ }^{5}$. However, the structures involved and the mechanisms by which changes occur have not been elucidated in patients with RA. 
Standard magnetic resonance imaging (MRI) and MR arthrography of the MTP joints have been used to describe anatomical detail, identify tears in the capsule and plantar plate, and facilitate surgical planning to ease MTP joint pain and instability in subjects without $\mathrm{RA}^{6,7,8,9}$. Controversy about the appearance of the distal insertion has been highlighted, in which a hyperintense region has been interpreted as evidence for a tear ${ }^{6}$. Conversely, MR arthrography of MTP joints in cadavers and in subjects without RA has demonstrated improved visualization and delineation of tears in the fibrous capsule and plantar plate ${ }^{10,11,12}$, but the technique has not been applied in patients with RA.

A recent MRI study has demonstrated that focal deficiencies attributed to plantar plate pathology of the lesser (second to fifth) MTP joints are common in the forefoot of patients with RA and associated with synovitis, bone edema, and bone erosion ${ }^{13}$.

Our primary aim in this exploratory study was to compare the clinical, biomechanical, and plain radiography findings with MR arthrography at the lesser MTP joints in patients with RA. A secondary aim was to compare standard unenhanced MR with MR arthrography in imaging the capsule and plantar plate of the lesser MTP joints in RA.

\section{MATERIALS AND METHODS}

Recruitment of patients. Local research and ethical approval was received and written consent was obtained from all participants. Consecutive patients diagnosed with RA, according to the 1987 American College of Rheumatology revised criteria for $\mathrm{RA}^{14}$ (all patients were diagnosed prior to the 2010 RA classification criteria ${ }^{15}$ ), and having pain on the plantar aspect of their lesser MTP joints were recruited between June 2010 and May 2011. The first MTP joint was not imaged because of the different anatomy and pattern of pathological changes compared to the lesser MTP joints. Patients were excluded if they had a diagnosis of diabetes, peripheral vascular disease, or peripheral neuropathy; a history of forefoot surgery; or contraindications to having an MRI scan and injection of the contrast agent.

Clinical, biomechanical, and radiography measures. Demographic data were recorded for each patient. In order to quantify current disease activity, a Disease Activity Score (DAS44) was used ${ }^{16}$. A 100-mm visual analog scale (VAS) score, with the anchors "no pain" and "worst pain imaginable," was recorded for current pain across the plantar MTP joint area ${ }^{17}$, and patients completed the Leeds Foot Impact Scale (LFIS), a self-completed foot health outcome tool for RA ${ }^{18}$. The LFIS has 51 items in 2 domains to assess impairment and footwear (LFIS ${ }_{\mathrm{IF}}$ ), and activity and participation $\left(\right.$ LFIS $\left._{\mathrm{AP}}\right)$. Platto's Structural Index was completed to quantify the extent of forefoot structural deformity ${ }^{19}$. The presence of plantar callus and subluxation at each lesser MTP joint was recorded by an experienced clinician (HJS). The EMED pressure platform (Novel GmbH, Munich, Germany) was used to measure barefoot peak pressure $(\mathrm{kPa})$ at each lesser MTP joint. Measurements were undertaken using the second step method and the average of 3 readings was recorded for each patient. Gait velocity was measured using the GAITRite instrumented walkway (GAITRite, CIR Systems Inc., Haverton, PA, USA). Standard antero-posterior radiographs were taken to identify the radiographic damage at each lesser MTP joint, and radiographs were read by an experienced consultant rheumatologist (PSH) using the Larsen score ${ }^{20}$.

MRI. The more symptomatic forefoot was imaged using a 3T Verio scanner (Siemens Healthcare, Erlangen, Germany) with an 8-channel or 15-channel radio frequency knee coil. The patient was lying in a supine position with the knee flexed; the forefoot was placed within the coil. Proton density fat-suppressed sagittal sequences [turbo spin echo (TSE), field of view (FOV) 130, acquisition time (TA) 6 min, repetition time (TR) $\sim 5 \mathrm{~s}$, echo time (TE) $34 \mathrm{~ms}, 1.5 \times 0.5 \times 0.3 \mathrm{~mm}$ resolution] and short axis sequences (TSE, FOV 130, TA $7 \mathrm{~min}$, TR $4 \mathrm{~s}$, TE $36 \mathrm{~ms}, 1.1 \times 0.5 \times 0.3 \mathrm{~mm}$ resolution) that had been optimized to demonstrate the plantar plate and joint were acquired through the lesser MTP joints prior to arthrography. Standard flip-angles for spin-echo images were used $\left(90^{\circ} / 180^{\circ}\right)$. Intraarticular contrast agent injection was performed by an experienced musculoskeletal radiologist (PO'C). Under ultrasound guidance, local anesthetic followed by $0.5-1.0 \mathrm{ml}$ of $2 \mathrm{mmol} / \mathrm{l}$ dimeglumine gadopentetate was injected into the dorsum of each of 2 lesser MTP joints. Standard MRI were reviewed by a radiologist prior to injection of the contrast agent; MTP joints were chosen where it was unclear whether plantar plate pathology was present, and where possible, injecting contrast agent into neighboring MTP joints was avoided Joints were excluded if the standard MR revealed that the plantar plate was absent or if subluxation or dislocation would prevent reliable intraarticular injection. Sagittal and short-axis T1-weighted fat-suppressed sequences (spin echo, FOV 150, TA $6 \mathrm{~min}$, TR $\sim 0.7 \mathrm{~s}$, TE $11 \mathrm{~ms}, 2.0 \times 0.6 \times 0.4 \mathrm{~mm}$ resolution) were subsequently acquired through the lesser MTP joints.

MR images were read prospectively by 2 experienced musculoskeletal radiologists (RJH and $\mathrm{AJG}$ ) and consensus was reached. Unenhanced images were initially assessed for deficiencies in the plantar plate (including medial and lateral insertions) or capsule, or absence of the plantar plate. In addition, the presence of central high signal intensity at the distal insertion was also recorded ${ }^{6}$. Subsequently, contrast agent extravasation was assessed on the arthrographic images. MR arthrographic abnormalities at the lesser MTP joints were ordered into 4 grades: (1) no pathology (Figure 1A, 1B); (2) extravasation of contrast agent into the adjacent soft tissues (interpreted as a capsule tear ${ }^{8,11}$; Figure 1C, 1D); (3) extravasation of contrast agent into the flexor digitorum longus tendon sheath (interpreted as a plantar plate tear $\left.{ }^{6,11}\right)$; and (4) extravasation of contrast agent into both the surrounding soft tissues and the flexor tendon sheath (Figure 2).

Statistical methods. Data were entered onto the Statistical Package for the Social Sciences (SPSS version 18). Simple descriptive statistics were reported for all demographic and clinical measures and plantar plate pathology. Mean (SD) scores were reported for interval level data, and median [interquartile range (IQR)] for ordinal data. Standard unenhanced MR images were compared with MR arthrography for sensitivity, specificity, and accuracy. Spearman's correlation coefficient was used to measure the association between MR arthrography abnormalities at the lesser MTP joints and clinical, biomechanical, and radiographic findings $(n=15)$. The joint with the highest category of pathology identified by MR arthrography was chosen per patient for analysis. The level of substantive association was identified at $\rho>0.3$. For this exploratory study no formal sample size calculations were performed, and no corrections for multiple comparisons have been made; significance values are presented as guidelines only.

\section{RESULTS}

Demographic, disease characteristics, patient-reported measures, forefoot deformity scores, gait measures, and radiographic scores are given in Table 1.

MR arthrography. MR arthrography was performed on 30 lesser MTP joints: 8 second MTP joints, 10 third MTP joints, 8 fourth MTP joints, and 4 fifth MTP joints. Contrast agent was not successfully injected into 2 joints and therefore these were excluded from subsequent analysis.

Correlation of MR arthrography-reported pathology with disease characteristics. The associations between MR arthrography abnormalities at the lesser MTP joints in patients with RA and clinical, biomechanical, and radio-

Personal non-commercial use only. The Journal of Rheumatology Copyright @ 2012 . All rights reserved. 


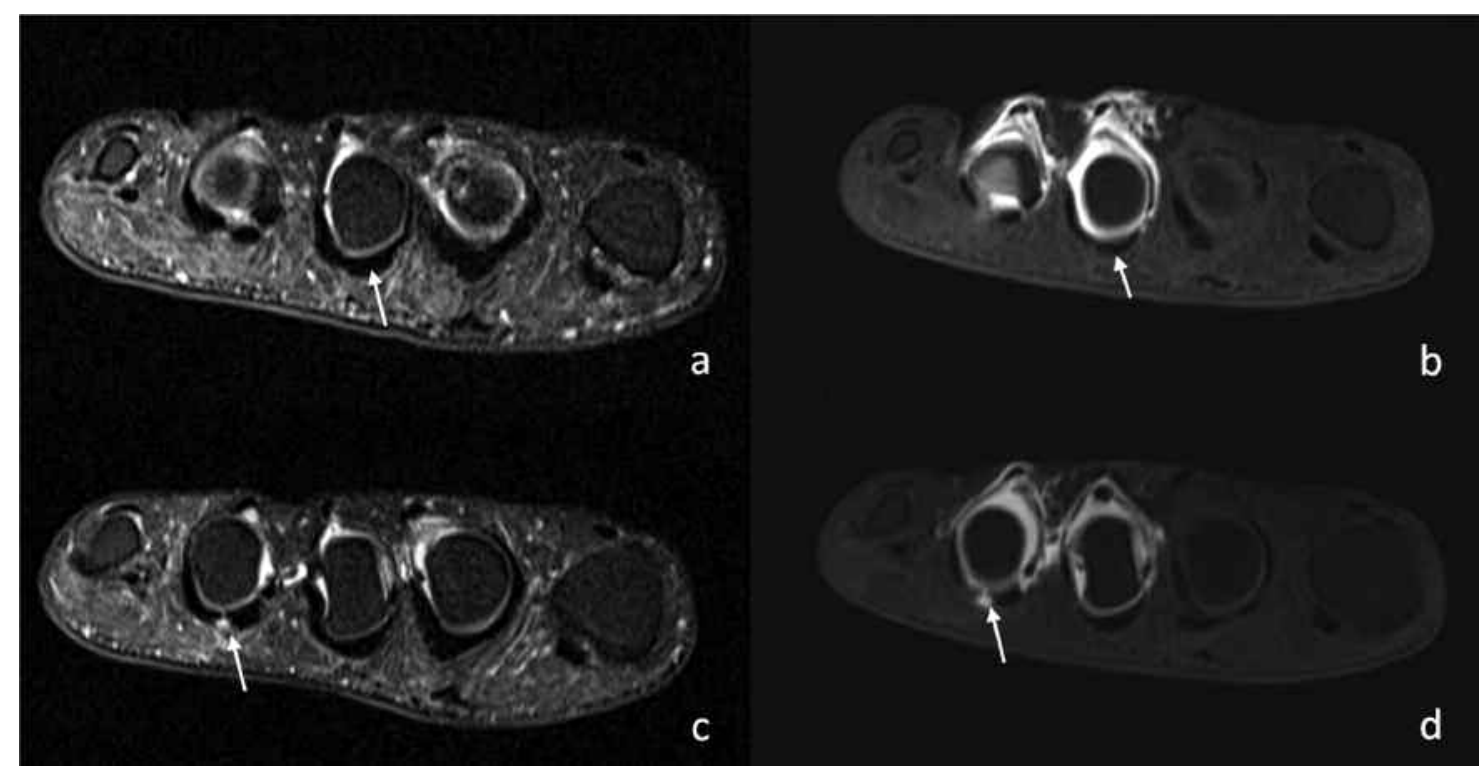

Figure 1. Pre (a) and post (b) contrast agent short-axis images of the third metatarsophalangeal (MTP) joint demonstrating intact capsule and plantar plate (arrows). Precontrast agent short-axis image (c) of the fourth MTP joint of the same patient demonstrating a capsular tear (arrow), which is confirmed in the postcontrast agent T1 short-axis image (d) with extravasation into the soft tissue (arrow).

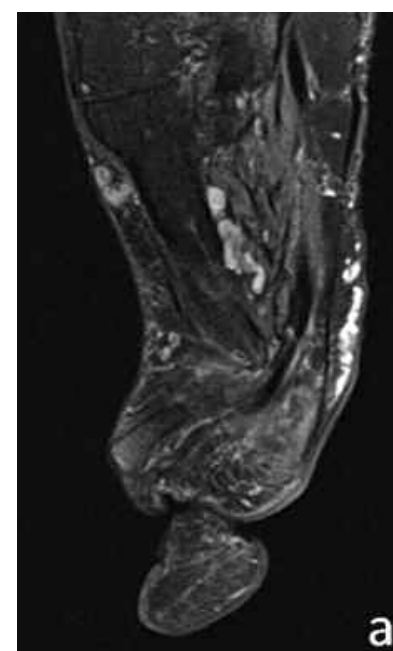

a

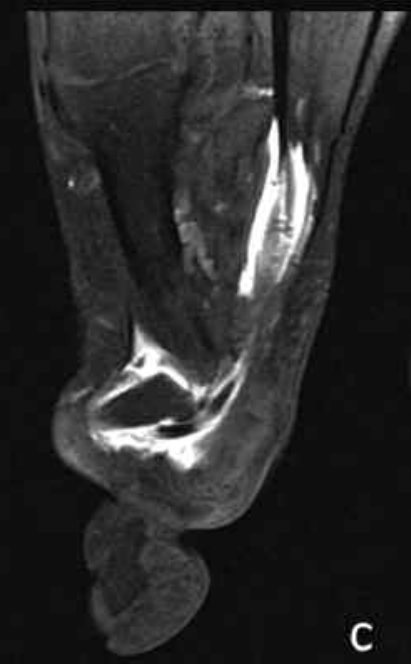

b

Figure 2. Precontrast agent sagittal and short-axis images (a) and (b) showing absent plantar plate and laterally displaced flexor tendon (arrow). Post-contrast agent $\mathrm{T} 1$ sagittal (c) and short-axis (d) images showing extravasation of contrast agent into the soft tissues and flexor tendon sheath (arrow) confirming an absent plantar plate. 
Table 1. Demographic, clinical, biomechanical, and radiographic variables.

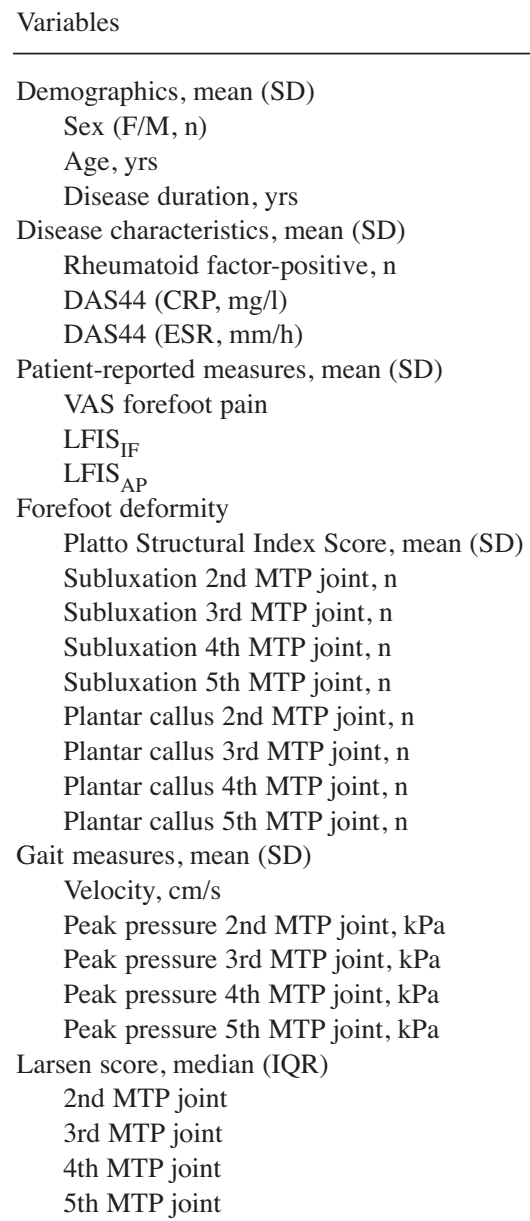

$$
\begin{gathered}
11 / 4 \\
29.4(9.8) \\
7.5(9.0 ; \text { range } 0.2-27) \\
11 \\
2.71(0.62) \\
2.83(0.68) \\
37.7(27.2 \text {; range } 8-95) \\
12.4(2.5 ; \text { range } 7-16) \\
14.9(8.7 \text {; range 3-30) }
\end{gathered}
$$

$6.0(4.1)$
8
8
8
4
6
1
0
1

$95.4(29.9)$

$730.9(387.6)$

$531.4(211.8)$

$248.0(76.5)$

$313.1(281.75)$

$1(0-4)$

$1(0-4)$

$1(0-3)$

$2(0-3)$

DAS: Disease Activity Score; CRP: C-reactive protein; ESR: erythrocyte sedimentation rate; VAS: visual analog scale; LFIS $_{\mathrm{IF}}$ : Leeds Foot Impact

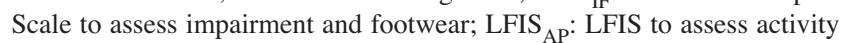
and participation; MTP: metatarsophalangeal; kPa: barefoot peak pressure; IQR: interquartile range.

graphic variables are given in Table 2. MR arthrography abnormalities were associated with disease duration, forefoot deformity (Platto's forefoot Structural Index score), Larsen score, plantar callus, clinician reported subluxation, and peak plantar pressure.

Plantar plate pathology seen on standard MR imaging and MR arthrography. On standard MR images, 4 plantar plates were absent and subsequent MR arthrography revealed extravasation of contrast agent into the surrounding soft tissue and flexor tendon, confirming a tear in the plantar plate in all 4.

Focal deficiencies were seen in 13 plantar plates $(50 \%)$ on standard MR with high signal at the insertion in 10 of these. MR arthrography confirmed deficiencies in 10 plantar plates, extravasation of contrast agent was seen in the surrounding soft tissue of 6 , confirming a tear in the capsule,

Table 2. Spearman's correlation coefficients between magnetic resonance arthrography-reported pathology at the lesser metatarsophalangeal joints in patients with rheumatoid arthritis and clinical, biomechanical, and radiographic variables.

\begin{tabular}{lrcc}
\hline Variables & \multicolumn{1}{c}{$\mathrm{r}_{\mathrm{s}}$} & $\mathrm{p}$ & $\mathrm{n}$ \\
\hline Disease duration & 0.728 & 0.001 & 15 \\
VAS & -0.122 & 0.332 & 15 \\
LFIS $_{\mathrm{IF}}$ score & -0.162 & 0.282 & 15 \\
LFIS $_{\mathrm{AP}}$ score & -0.002 & 0.497 & 15 \\
Platto's FF Structural Index score & 0.535 & 0.020 & 15 \\
Gait velocity & -0.200 & 0.237 & 15 \\
Larsen score & 0.818 & 0.000 & 15 \\
Callus present & 0.523 & 0.023 & 15 \\
Subluxation present & 0.486 & 0.033 & 15 \\
Peak pressure & 0.629 & 0.006 & 15 \\
\end{tabular}

VAS: visual analog scale; LFIS $_{\mathrm{IF}}$ : Leeds Foot Impact Scale to assess impairment and footwear; LFIS $_{\mathrm{AP}}$ : LFIS to assess activity and participation; FF: forefoot.

and extravasation of contrast agent was seen in the flexor tendon of only 4 , confirming a true plantar plate tear ${ }^{11}$. Two plantar plates showed no extravasation of contrast agent on arthrography (contrast agent was not successfully injected into 1 joint); both demonstrated high signal at the insertion on standard MR.

Thirteen plantar plates appeared intact on standard MR images; arthrography confirmed that 8 were intact, with no contrast agent extravasation. High signal was seen at the insertion of all 8. MR arthrography showed capsular tears in 3 with extravasation of contrast agent into the surrounding soft tissue and a tear of the plantar plate in 1 (contrast agent was not successfully injected into 1 joint); high signal was seen in 2 of the torn capsules and in the torn plantar plate on standard MR.

Absence or focal defect of the plantar plate seen on standard MR in 28 lesser MTP joints had a sensitivity of $78 \%$ (95\% CI 52-93), specificity of 90\% (95\% CI 54-99), and accuracy of $82 \%$ for detecting a tear, compared to MR arthrography (Table 3). High signal at the insertion on standard MR had a sensitivity of $80 \%$ (95\% CI 56-93), specificity of $15 \%$ (95\% CI 5-36), and accuracy of $43 \%$ for detecting a tear.

\section{DISCUSSION}

To our knowledge, this is the first study to report the use of MR arthrography for the evaluation of the painful forefoot in patients with RA and to compare MR arthrography with clinical, biomechanical, and plain radiography findings in a cross-sectional group of patients with RA and forefoot pain. We have demonstrated that capsule and plantar plate pathology are common in the painful forefoot of patients with RA and may be associated with features of disease and deformity at the lesser MTP joints.

Previous studies in cadaveric specimens and subjects Personal non-commercial use only. The Journal of Rheumatology Copyright @ 2012 . All rights reserved. 
Table 3. Absence or focal defect of the plantar plate seen on standard magnetic resonance imaging (MRI) compared to contrast agent extravasation on MR arthrography in 28 lesser MTP joints in patients with RA.

\begin{tabular}{lccc}
\hline & $\begin{array}{c}\text { MR Arthrography } \\
\text { Contrast Agent } \\
\text { Extravasation }\end{array}$ & $\begin{array}{c}\text { No Contrast Agent } \\
\text { Extravasation }\end{array}$ & Total \\
\hline $\begin{array}{l}\text { Standard MR } \\
\text { Absence or focal } \\
\text { defect of plantar plate }\end{array}$ & 14 & 1 & 15 \\
$\begin{array}{l}\text { Normal plantar plate } \\
\text { Total }\end{array}$ & 4 & 9 & 13 \\
\hline
\end{tabular}

without RA have compared standard MR imaging with MR arthrography in diagnosing soft tissue pathology at the lesser MTP joints ${ }^{6,10,11}$. These studies have suggested that MR arthrography improves visualization of the structures and delineates capsule tears from plantar plate tears. Results from our study in RA suggest that when compared with MR arthrography, standard MR imaging is specific but only moderately sensitive for diagnosing plantar plate tears based on the absence or focal defect of the plate. Previous MRI research has suggested that increased signal intensity may also be seen immediately adjacent to the proximal phalanx insertion where there is no tear present ${ }^{6}$. Our findings are consistent with this and support the contention that when compared with MR arthrography, the presence of high signal at the plantar plate insertion on standard MR in RA is not specific for plantar plate or capsular tear, indicating that high signal seen on standard MR at the plantar plate insertion is not a useful sign. While our results give an indication of the diagnostic utility of standard MR, the inclusion of more than 1 joint per patient may have introduced some inaccuracy to the estimates; the findings therefore would need to be repeated in a larger sample with formal adjustment for clustering of joints within individuals.

In our study, MR arthrography abnormalities were not associated with VAS forefoot plantar pain or either domain of the LFIS. Our sample comprised patients with low disease activity (few tender and swollen joints) who were in receipt of foot healthcare from a specialist rheumatology foot health clinic. Further, patients were asked to report current forefoot pain and complete the LFIS while thinking about their feet at the moment, hence pain was evaluated for only a single moment in time and may not be a true reflection of previous pain, which may have contributed to the cumulative MTP joint pathology. We did not differentiate between pain at individual lesser MTP joints, and therefore further work is needed to establish whether there is a correlation between pain at individual MTP joints and MR arthrography abnormalities at the same joint. Our study may be limited because we assessed only plantar plate and capsule pathology at the lesser MTP joints; pathology of other soft tissue structures may contribute to pain and deformity.
Lesser MTP joint capsule and plantar plate pathology are substantively associated with disease duration, biomechanical changes in the forefoot, and radiographic damage in patients with RA. However, a limitation of the study is the small sample size $(n=15)$, which prevented a full multivariable analysis to evaluate whether features of disease progression and forefoot deformity were related to MR arthrography abnormalities independently of each other. A further limitation of our study is the lack of a healthy control group without foot pain and RA. Given the invasive nature and the potential for adverse reactions when using contrast agent, it was deemed unnecessary and inappropriate to inject contrast agent into healthy individuals at this exploratory stage, especially because our aims were to compare imaging modalities as they relate to lesser MTP joint pathologies specifically in patients with RA. Despite these limitations, our exploratory study will help inform larger controlled cross-sectional studies and longitudinal followup studies of patients with RA and forefoot pain to help elucidate causality.

MR arthrography has demonstrated that capsule and plantar plate pathology at the lesser MTP joints in patients with RA may be associated with features of disease progression and forefoot deformity. This may have important implications in assessing the progression of forefoot damage and understanding the causes of symptoms in the painful forefoot of patients with RA.

\section{ACKNOWLEDGMENT}

The authors thank Carole Burnett and Robert Evans from the NIHR Leeds Musculoskeletal Biomedical Research Unit for conducting the patient MRI.

\section{REFERENCES}

1. Grondal L, Tengstrand B, Nordmark B, Wretenberg P, Stark A. The foot: still the most important reason for walking incapacity in rheumatoid arthritis: Distribution of symptomatic joints in 1,000 RA patients. Acta Orthop 2008;79:257-61.

2. Spiegel TM, Spiegel JS. Rheumatoid arthritis in the foot and ankle - Diagnosis, pathology, and treatment. The relationship between foot and ankle deformity and disease duration in 50 patients. Foot Ankle 1982;2:318-24.

3. Keenan MA, Peabody TD, Gronley JK, Perry J. Valgus deformities of the feet and characteristics of gait in patients who have rheumatoid arthritis. J Bone Joint Surg Am 1991;73:237-47.

4. Jaakkola JI, Mann RA. A review of rheumatoid arthritis affecting the foot and ankle. Review. Foot Ankle Int 2004;25:866-74.

5. Stainsby GD. Pathological anatomy and dynamic effect of the displaced plantar plate and the importance of the integrity of the plantar plate-deep transverse metatarsal ligament tie-bar. Ann R Coll Surg Engl 1997;79:58-68.

6. Yao L, Do HM, Cracchiolo A, Farahani K. Plantar plate of the foot: Findings on conventional arthrography and MR imaging. AJR Am J Roentgenol 1994;163:641-4.

7. Umans HR, Elsinger E. The plantar plate of the lesser metatarsophalangeal joints: Potential for injury and role of MR imaging. MRI Clin N Am 2001;9:659-69, xii.

8. Powless SH, Elze ME. Metatarsophalangeal joint capsule tears: An analysis by arthrography, a new classification system and surgical management. J Foot Ankle Surg 2001;40:374-89. 
9. Gregg J, Silberstein M, Schneider T, Marks P. Sonographic and MRI evaluation of the plantar plate: A prospective study. Euro Radiol 2006;16:2661-9.

10. Mohana-Borges AV, Theumann NH, Pfirrmann CW, Chung CB, Resnick DL, Trudell DJ. Lesser metatarsophalangeal joints: Standard MR imaging, MR arthrography, and MR bursography initial results in 48 cadaveric joints. Radiology 2003;227:175-82.

11. Kier R, Abrahamian H, Caminear D, Eterno R, Feldman A, Abrahamsen T, et al. MR arthrography of the second and third metatarsophalangeal joints for the detection of tears of the plantar plate and joint capsule. AJR Am J Roentgenol 2010;194:1079-81.

12. Blitz NM, Ford LA, Christensen JC. Second metatarsophalangeal joint arthrography: A cadaveric correlation study. J Foot Ankle Surg 2004;43:231-40.

13. Siddle HJ, Hodgson RJ, Redmond AC, Grainger AJ, Wakefield RJ, Pickles DA, et al. MRI identifies plantar plate pathology in the forefoot of patients with rheumatoid arthritis. Clin Rheumatol 2012;31:621-9.

14. Arnett FC, Edworthy SM, Bloch DA, McShane DJ, Fries JF, Cooper NS, et al. The American Rheumatism Association 1987 revised criteria for the classification of rheumatoid arthritis. Arthritis Rheum 1988;31:315-24.
15. Aletaha D, Neogi T, Silman AJ, Funovits J, Felson DT, Bingham CO 3rd, et al. 2010 rheumatoid arthritis classification criteria: An American College of Rheumatology/European League Against Rheumatism collaborative initiative. Ann Rheum Dis 2010;69:1580-8

16. van der Heijde DM, van 't Hof MA, van Riel PL, Theunisse LA, Lubberts EW, van Leeuwen MA, et al. Judging disease activity in clinical practice in rheumatoid arthritis: first step in the development of a disease activity score. Ann Rheum Dis 1990;49:916-20.

17. Budiman-Mak E, Conrad KJ, Roach KE. The Foot Function Index: A measure of foot pain and disability. J Clin Epidemiol 1991;44:561-70.

18. Helliwell P, Reay N, Gilworth G, Redmond A, Slade A, Tennant A, et al. Development of a foot impact scale for rheumatoid arthritis. Arthritis Rheum 2005;53:418-22.

19. Platto MJ, O'Connell PG, Hicks JE, Gerber LH. The relationship of pain and deformity of the rheumatoid foot to gait and an index of functional ambulation. J Rheumatol 1991;18:38-43.

20. Larsen A, Dale K, Eek M. Radiographic evaluation of rheumatoid arthritis and related conditions by standard reference films. Acta Radiol Diagn (Stockh) 1977;18:481-91. 